\title{
Abstracts of the Malaysian Medical Students Summit, MMSS, 2015
}

01. Major postpartum haemorrhage secondary to acute complete uterine inversion, a case report.

C S Chean ${ }^{1}$, Peter Liao ${ }^{1}$, S Shaman, S S Poon ${ }^{1}$, A Soltan ${ }^{2}$

${ }^{1}$ Faculty of medicine, University of Liverpool, ${ }^{2}$ Liverpool Women's Hospital

Introduction a objectives: Obstetric haemorrhage is one of the leading causes of maternal death in developed and developing countries. Acute uterine inversion is a rare obstetric emergency, estimated to occur in 1 of 3500 to 5000 deliveries. Patient/Method A 35-year-old lady ( $\mathrm{C}_{2} \mathrm{P}_{2}$ ) was admitted for induction of labour at $41+3$ weeks as per local protocol for the management of postdates pregnancies. She gave a history of previous postpartum haemorrhage (PPH) following her first pregnancy elsewhere. The plan was active management of third stage with routine precautions (e.g. IV access, blood sample for group and save etc.) to be taken in case recurrent PPH. In theatre, the uterine inversion was corrected manually with no difficulty immediately as the patient was anaesthetised. However, she continued to suffer major (atonic) PPH and the urine started to become blood stained raising concerns about DIC. Results a Discussions: The switching off of volatile anaesthetics and insertion of Bakri Balloon was successful in controlling this patient's major PPH. On day 2 postoperatively, she seemed to have recovered relatively quickly and the Bakri Balloon was removed. The uterus was well contracted with minimal lochia and the patient was discharged home on day 3 postnatal with a healthy baby. Conclusion: Acute uterine inversion is a rare but severe condition and all staff should be aware of PROMPT (Practical Obstetric Multi-Professional Training) and the management of PPH as described in the RCOG green-top guideline No. 52 as delay in treatment is associated with high mortality and morbidity.

02.

Clinical Audit on Diagnostic at Treatment Recommendation of Dry Eyes in St. Paul's Eye Unit

S Chean ${ }^{1}$, M Aswin ${ }^{1}$, S S Poon ${ }^{1}$, A Sajjad ${ }^{1}$

${ }^{7}$ Faculty of Medicine, University of Liverpool, ${ }^{2}$ St. Paul's Eye Unit, Royal Liverpool and Broadgreen University Hospital

objective: Clinical audit of diagnosis and treatment methodologies of dry eyes in St. Paul's eye unit compared to the standard recommendations of Dry Eyes Workshop (DEWS) report 2007 Methods: Data are collected from 18 dry eye patients for a total period of 3 months. Patient demographics are analysed and the diagnostic methodologies used in St. Paul's Eye Unit were reviewed. The severity of dry eye of every patient in this audit is then graded according to the standard. Their respective treatmen provided in St. Paul's are then compared against the standard treatment recommendations. Results: The mean age of patients $(\mathrm{N}=18)$ is $70.5(\mathrm{SD}=12.63)$ whereas all patients in this audit are female. Patients are then divided into subgroups of Primary Sjogren's syndrome dry eye, Secondary Sjogren's syndrome dry eye and non- Sjogren's syndrome dry eye. Most patients (55.6\%) suffer from Grade 3 severity dry eyes. One-third (33.3\%) of patients have adequate treatment which correlates with their dry eye severity grading.

Discussion: The standard diagnostic protocol recommended by DEWS report should be followed. Most practitioners in St. Paul Eye Unit appear to be more conservative in their treatment of dry eye patients of varying severity. However, the standard guideline noted that treatment recommendations can be modified by practitioners on the basis of their clinical experience and individual patient profile.

Conclusion: The standard guidelines of dry eye diagnosis and management is of considerable clinical utility and it is advised to be used by practitioners based on each individual patient profile and clinical experience for the best interests of the patients.

03. Clostridium Perfringens Enterotoxin (CPE) in Clostridium Perfringens Gastroenteritis: A review of recent evidence Goh JE, Lee LH

leffrey Cheah School of Medicine and Health Sciences, Monash University Malaysia.

Introduction: Clostridium Perfringens is referred as a worldwide pathogenic microorganism in nature as well as in humans. Its associated type A food-poisoning has been classified among the most widespread foodborne gastroenteritis diseases in the developed countries globally. Objective: To review existing and novel aspects of $C$. Perfringens, its toxin Clostridium Perfringens Enterotoxin (CPE) and its associated type A foodborne illness. Methodology: Pubmed, Science Direct and Elsevier were systematically searched using "Clostridium Perfringens", "Clostridium Perfringens Enterotoxin" and "Clostridium Perfringens type A Gastroenteritis" as keywords. Fifty articles published in the past 10 years were selected to be reviewed on prevalence and sources of infection; identification of bacteria and its toxin CPE; treatment and public health measures to curb this issue and CPE as potential medical application. Results: Substantial experimental and epidemiologic studies have provided rigid evidence on the role plays by CPE as the major virulence factor responsible for the pathogenesis of $C$. Perfringens type A gastroenteritis. Significant advancement on the knowledge available on $C$. Perfringens and its associated gastroenteritis has been made. Discussion: Public health measures in preventing further outbreak cases from happening are especially important and much attention is given towards effective disinfection routines and proper food handling practices which aid in removing the presence of any bacteria. Conclusion: Many challenges remain for researchers, including the physiology, genetics and mechanism of action of different cpe positive strains and the reassessment of the current diagnostic tools to more explicit methods in identifying C. Perfringens type A food-poisoning outbreak. Besides that, there is a need for more in-depth views on the sources and reservoirs of $C$. Perfringens cpe-positive strains for better control of this organism in food.

04. The role of group II mGluRs in synaptic transmission and plasticity at the CA2 region of the hippocampus

Jonathan Lee, Chuo Min

Introduction: Group II metabotropic glutamate receptors (mGluR) have been known to depress neuronal transmission via inhibition of neurotransmitter release at glutamatergic synapses. They can also mediate long term depression in several regions of the brain including the lateral amygdala and striatum. However, their roles in the hippocampus, particularly the cornu ammonis 2 (CA2) region is largely unclear. Objectives and Methods: We have used electrophysiological techniques on wildtype and mCluR2 deficient rats in combination with group II mGluR modulating drugs to tease apart the physiological functions of group I mGluRs on the temporo ammonic pathway input synapse to the CA2. Results and Discussion: Using DCG-IV (group II mGluR agonist), we have identified that activation of group II mGluRs leads to depression of synaptic transmission in the TAP-CA2 synapse in a dose-dependent manner. Washout of DCG-IV resulted in LTD which indicates that group II mGluRs can mediate LTD in the same region. Using LY 541850 (mGluR2 agonist, mGluR3 antagonist) on wildtype rats, DCG-IV on mGluR2 deficient rats, we have further evidence that activation of mGluR $2 / 3$ alone can induce significant depression of synaptic response as well as induce LTD. Using these drugs in combination, we have also 
established that mGluR2 and mGluR3 may have synergistic effects on one another, potentiating depression and lowering the threshold for LTD induction. Conclusion: These results suggest important roles of group II mGluRs as regulators of CA2 activity which may have significant implications in explaining the mechanism behind the clinically beneficial (eg. neuroprotective/anxiolytic) effects of group II mGluR modulation. These episodic memory in the hippocampus

05. Pain, itch and quality of life after Herpes zoster: A web-based prospective study

Albert JM van Wijck ${ }^{1}$, Yueting Kew ${ }^{2}$

${ }^{7}$ Pain Clinic, Department of Anesthesiology, UMC Utrecht, The Netherlands, ${ }^{2}$ Faculty of Medicine, University of Malaya, Kuala Lumpur, Malaysia

Introduction: Pain and itch are the most common complication in herpes zoster ( $\mathrm{HZ}$ ) patients and are difficult to treat. However long-term impact on quality of life (QOL) is not known. Aims: To measure the incidence of pain and itch after $\mathrm{HZ}$ and the impact on QOL Methods: We performed an observational prospective study in patients above 50 years with $\mathrm{HZ}$. Participants were recruited by internet and by general practitioners within 7 days after onset of the rash. Follow-up was web-based for pain, itch and quality of life at 2 weeks an 1, 3, 6 and 12 months. There were no restrictions in treatment. Significant pain or itch was defined as a score . 30 on a $100 \mathrm{~mm}$ VAS. Results: A web-based prospective study was feasible in the 50+ population. We enrolled 661 patients with a follow-up of at least one month. Mean age was 65 years (range 50-96). Presence of PHN reduced Quality of Life to a large degree. Results are displayed in figure 1 and 2 . Conclusion: Although the risk of long lasting severe PHN is low, the burden of disease is high.

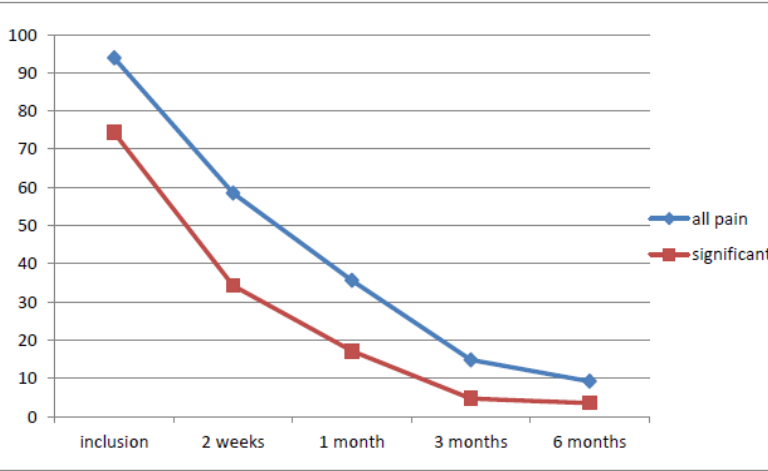

Figure 1: Percentage of patients with pain

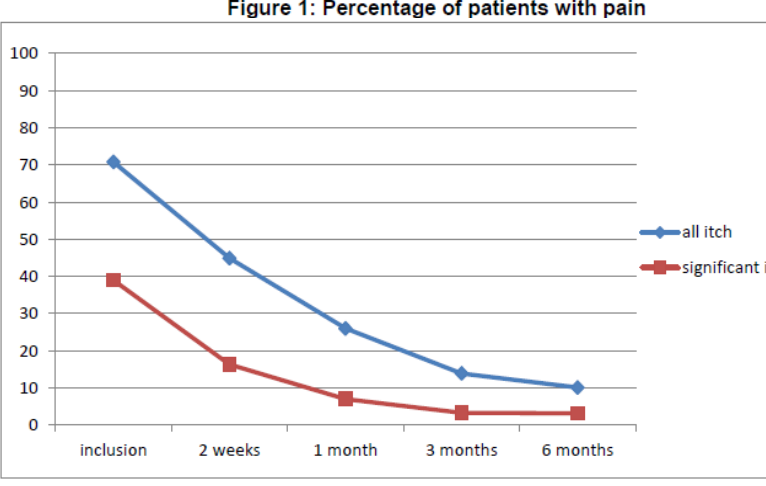

Figure 2: Percentage of patients with itch
The Frequency of Neuropathic Pain in Low Back Pain Patients Attending a Tertiary Spine Clinic

Cheng Yin Tan' ${ }^{1}$, Yueting Kew' ${ }^{1}$, Sue Sien Thang', Leong Hooi Tan', Yvonne Khaii Khoo', Chong Jing', Jun $\mathrm{Ni}$

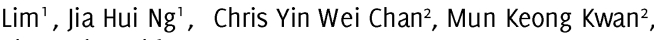
Khean Jin Goh

Division of Neurology, Department of Medicine and ${ }^{2}$ Department of Orthopaedic Surgery, University of Malaya, Kuala Lumpur, Malaysia

Aim: Chronic back pain may have a combination of both nociceptive and neuropathic pain components. The objective of the study is to evaluate the neuropathic pain component in a group of outpatients with low back pain Methods: The painDETECT neuropathic pain screening questionnaire was used to identify pain mechanisms in a cohort of patients seen at the Spine Clinic, University of Malaya Medical Centre, Kuala Lumpur. Pain mechanisms were classified as nociceptive, unclear and neuropathic and were correlated with demographic factors, associated diseases, underlying diagnosis for low back pain as well as pain severity (numerical rating scale), disability (Roland Morris Disability Questionnaire) and anxiety/depression (Hospital Anxiety and Depression Scale). Results: of the 210 patients recruited, $26(12.4 \%)$ had neuropathic pain, $45(21.4 \%)$ were unclear and $139(66.2 \%)$ were likely nociceptive pain. Subjects with neuropathic pain were more often women, more likely to have leg pain and to have the pain radiate below the knee. There were no differences between ethnic groups, associated diseases, underlying cause or duration of back pain. Patients with neuropathic pain had significantly more severe average ( 6.8 vs. $4.7, P<0.001$ ) and highest ( 8.4 vs. $5.6, P<0.001)$ pain score over the last 4 weeks. They also had higher RMDQ scores (16.4 vs, 10.0, $\mathrm{P}<0.001$ ) and more of them had anxiety $(42.3 \%$ vs. $12.2 \% ; \quad P<0.001)$. Conclusions: The frequency of neuropathic pain was $12.4 \%$. Low back pain with neuropathic pain component was more severe and was associated with greater disability and more anxiety in patients.

07. How Does Educational Theory Influence the Teaching of Clinical Skills

Y W Liao

University of Liverpool, Liverpoo

Introduction: From palpating to auscultating, doctors use these clinical skills in everyday practice to help diagnose patients. Hence, there is no surprise that the teaching of clinical skills is a fundamental pillar of every medical school. objective: The objective of a contemporary medical education aims to produce medical professionals with multiple competencies, one of which is clinical skills. This paper aims to deconstruct the concept of teaching clinical skills and seeks to discuss the types of theories used to teach clinical skills. By reviewing the fundamentals and history of each theory, this paper will then seek to uncover the education theories used in clinical skills sessions and understand the implications and effects of each theory. Method: A literature search was performed using online database (Pubmed and Scopus). To refine the search and ensure consistency, a specific set of inclusion and exclusion criteria was agreed on and applied for both databases. Results: A total of 20 papers were selected. Discussion: Four key education theories were examined and related to the teaching of clinical skills. These 4 theories are the curriculum theory, behaviourism, cognitivism, and constructivism. Every theory was related to the teaching of clinical skills and its effectiveness is then judged and evaluated. Conclusion: Education theories are not mutually exclusive and can be integrated into a clinical skills session more than one at a time. However, the choice of which theory to be integrated is highly dependent on the specific clinical skill being taught and its perceived effectiveness during the session.

\section{Self-Management of Gestational Diabetes Mellitus Y W Liao University of Liverpool, Liverpoo}


Introduction: The prevalence of Gestational Diabetes Mellitus (GDM) has been on the rise globally with studies stating that up to $14 \%$ of pregnancies are complicated due to diabetes and $3 \%---5 \%$ of that number is GDM. Hence, it is no surprise that GDM has become a particularly important topic of research within the specialty of obstetrics and gynecology. Objective: This pape seeks to investigate the feasibility of self--management of GDM by reviewing various papers recommending different self---management techniques, the effectiveness of self---managing GDM and if the patient is placed in any harm by choosing to self--- manage GDM. Method: A literature search was performed using online database (Pubmed and Scopus). A specific set of inclusion and exclusion criteria was applied to both databases. Only RCTs and systematic reviews were included. Result: 20 papers were selected. As the prevalence of GDM may differ according to ethnicity, the 20 papers originated from various countries to ensure a diverse sample size was taken into account. Discussion: A few areas were considered to determine the feasibility of self---management of GDM. 1) Patient adherence to self---monitoring of blood glucose --- Evidence shows that prope pharmacological intervention time is not significantly affected if blood glucose is recorded daily or less frequently. 2) Effectiveness of nutritional management - Studies have shown that proper die management is effective in managing and even preventing GDM. 3) Self-management techniques in an ambulatory setting - Various studies have recommended different ways to control GDM in an ambulatory setting. Conclusion: Based on current evidence, it is possible to self-manage GDM in an ambulatory setting with prope support from the health care team. This is especially important as GDM will last for the duration of the pregnancy hence self management is needed to ensure proper blood glucose levels are maintained.

09. Effect of Music Tempo on Blood Pressure, Heart Rate and Respiratory Rate among Medical Students in Melaka Manipal Medical College: A Single Blinded Randomised Controlled Trial

Aiman A, Amalina N, Malkit S, Salman B, Ikram K

Introduction: Music plays a complex role in human beings causing diverse physiological changes, including both neurological and cardio-respiratory aspects. Studies have shown that different music tempos have contrasting effect on the vitals. Objectives: The aim was to evaluate the effect of two types of music tempos on blood pressure, heart rate and respiratory rate among young adults in a medical college setting. Methods: The study was a single- blinded randomized controlled trial with sample size of 40 medical students, divided into two groups. Students were asked to listen to the songs for 7 minutes depending on which group they belonged: either fas tempo songs $2120 \mathrm{BPM}$ or slow tempo 52-108BPM. Blood pressure, heart rate and respiratory rate were measured pre and post intervention. Results: After listening to slow tempo songs, there were significant reductions in mean systolic blood pressure $(P$-value $=0.01$ ) and mean diastolic blood pressure $(P$ value $<_{0.01}$. However, there were no difference in mean systolic and diastolic blood pressure for fast tempo songs ( $P$-value $=0.07)$ and ( $P$-value $=0.23$ ) respectively. As for the heart rate, changes were insignificant ( $P$-value 0.05 for both). Per contra, the only significant difference for respiratory rate was for the fast tempo songs (P-value<0.01) Discussion: The results showed that slow tempo songs causes reduction in systolic and diastolic blood pressure. This is further supported by a study conducted by Siritunga et al that demonstrates reduction in mean systolic and diastolic blood pressure of subjects after listening to classical Indian music. Conclusion: In conclusion, slow tempo music can be used in bringing down blood pressure of patients or subjects where applicable.
10. Influence of Body Mass Index on Visual Reaction Time. A Cross-sectional Analytical Study.

Ngo Choon Wei

Introduction: Visual system faces the daunting task of rapidly processing an enormous range of information in everyday perception; identifying items, determine layouts and guide action to them. Humans need an effective visual system for everyday exploration and interaction, more so in medical personnel, whereby visual reaction is vital for every case management. objective: To determine the relationship between Body Mass Index (BMI) and Visual Reaction Time (VRT) in medical students. Methodology: Cross-sectional analytical study was done at Melaka Manipal Medical College between October-November 2014. We included 112 students without medical conditions including neurological disorders, cardiovascular disorders and diabetes. Subjects were asked to complete a standard questionnaire before having their height measured using calibrated measuring tape in Frankfort plane, and their weight measured using electronic weighing balance. Body Mass Index (BMI) was calculated using Quatelet Index, and categorized using Asian BMI. Visual Reaction time was recorded using validated Human Benchmark program. Results: Significant difference was seen in different groups in BMI, namely underweight, normal and overweight, with respect to visual reaction time. The other variables such as gender, ethnicity, caffeine consumption, fatigue level, exercise and handedness are not significantly associated with VRT.

Discussion: BMI was found to be linked with the nerve conduction velocity, small fiber neuropathy, alteration of nerve inhibition capacity and causing abnormalities in myelination due to high levels of adipokines. Extreme BMI especially underweight persons experience a dysregulation in the hormone secretion could result in cognitive disorders.

Conclusion: Underweight individuals have been shown to have prolonged median visual reaction time as compared to their normal and overweight BMl counterparts. Further study on this is needed as BMI may not correlate well with the body fat composition of subjects, for which we propose further study to use waist-hip ratio instead.

11. Effects of Energy Drinks on the Peak Expiratory Flow Rate (PEFR) among Medical Students of Different Races in Malaysia: A Randomized Controlled Trial

Adamlebbe Dulkifly, Nishalini Chandrasekaran, Hafez Amir Samia, Rowena Lau, Yong Yung Sze

Introduction: Energy drinks are commercially available drinks containing natural ingredients promoted by the manufacturers to improve performance, concentration, endurance and alertness. Several studies have proven that energy drinks have a significant effect on cardiovascular and cerebrovascular system. However, minimal studies have been done to study the effect of energy drink onrespiratory system (RS). One of the method to assess RS is peak expiratory flow rate (PEFR). objective: In this study, we aim to determine the effect of energy drink on PEFR in healthy young adults. Method: We performed a randomized, single-blinded study on a group of 40 young adults (20 males and 20 females). Participants were handed out questionnaires andthe drinks in an unlabeled styrofoamcup $(355 \mathrm{ml})$. Their initial pulse rates were counted for 60 seconds immediately after consuming the drink. After 30 minutes, their level of alertness were tested, peak flow rate and pulse rate were measured. Result: Consumption of the energy drink led to an increase in PEFR ( $p<0.05)$ compared to the placebo group. There was no significant difference in the mean of the immediate pulse between the two groups ( $p>0.05$ ). However, there was a significant increase in the pulse rate after 30 minutes of consuming the energy drink $(p<0.05)$. Additionally, there was an increase in perception of energy level after consumption of both drinks ( $p<0.05)$; which was more significant in those who consumed the energy drink compared to the placebo $(p<0.05)$. Conclusion: An increase in the Peak Expiratory Flow Rate (PEFR), 
pulse rate and perception of energy level was noted in young healthy individuals after consuming the energy drink.

12

\section{Parental Bonding and Self Esteem among Melaka Manipal} Medical College Students

Ong Jia Wen, Siti Nurr Syafeena Binti Rais, Nur Hanisah Binti Abdul Halim, Nurmardhati Abyan Binti Mad Shah ,Nurul Azleen Binti Abdul Aziz .

Melaka Manipal Medical College

Introduction: The quality of the parent-child relationship has been argued to have a significant impact on the competence, resilience, and well-being of all individuals. This cross-sectional study was conducted to study the relationship between parental bonding and self-esteem among medical students in Melaka Manipa Medical College. The sample comprised of 168 respondents from Batch 28 and Batch 29 MBBS students. The data was obtained by using Parental Bonding Instruments by Cordon Parker, Hilary Tupling and L.B. Brown and Rosenberg's Self-esteem Scale by D Rosenberg. Results: From the results, it is found that there is a significant relationship between parental bonding for both mother $(p=0.00014)$ and father $(p=0.00254)$ with self-esteem. Parents who are acceptance, approving, responsive and nurturing shows elevated perception of self-worth and competence among the students. In contrast, students subjected to less accepting parental practices (disapproving, unresponsive and uninterested) show low self-confidence, poor self- perception, and low selfesteem. Based on Parental Bonding Instrument, there are 4 types of parental styles namely affectionate constraint parenting (high care and high protection), affectionless control parenting (low care and high protection), optimal parenting (high care and low protection) and neglectful parenting (low care and low protection). Optimal parenting has shown a strong association with high self-esteem among the students. Conclusion: Parents should be optimum in their parenting, ie. High care but low protection so that their children will have higher self-esteem. In medical profession, high self-esteem leads to better performance and interpersonal success which has been regarded as the utmost important quality as it enhances participation in social welfare and healthcare management.

13. The Anxiolytic Effects of Lavender Scent Among Medical Students: A Randomized- Controlled Trial

Ong Li Wen, Muhammad Haziq, Muhammad Farid, Nantha Kumar

Introduction: Lavender (Lavandula angustifolia) is often recommended for stress relief and believed to possess anxiolytic effects. 0bjectives: To assess the efficacy of lavender in reducing anxiety among medical students. Methods: A single-blinded randomized-controlled trial was conducted among Melaka Manipal Medical College students.40 participants were stratified into female and male groups, and randomly assigned to intervention and control groups.Intervention group were asked to enter an enclosed darkroom diffused with lavender scent by using ceramic candle warmer with its upper compartment filled with lavende oil while control group were asked to enter another room with same setting except that lavender oil has been replaced by plain water.Anxiety was induced by providing visual and auditory stimuli in video form.20-item State Trait Anxiety Inventory(STAI-20) questionnaire was used to measure anxiety level.Pre and postintervention heart rate and blood pressure were noted.The raw data collected was processed using Microsoft Excel 2003,analysed using Epi Info7 for Windows(7.1.3) and GraphPad software.Results were recorded as frequencies, means,standard deviation, and $P$ values.A P value of, 0.05 is significant. Results: STAI-20 Score shows significant reduction in intervention group compared to the control group with $P$ value of 0.011 . Other results of change in systolic blood pressure, diastolic blood pressure and heart rate show no significant association between intervention and control group with $P$ value $<0.05$. Discussion: STAI-20 Score with $P$ value 0.011 indicates lavender significantly reduce anxiety among participants. Our result is consistent with study done by Kutlu et al
2008. However, our result shows no significant effect of lavender on blood pressure and heart rate. Conclusion: Our study shows significant effect of lavender scent on reducing anxiety among medical students. In further studies, we recommend a larger sample size and the use of other form of anxiety induced stimulus.

\section{Ability of Noggin Gene Therapy to Reduce Heterotopic Ossification in Fibrodysplasia 0ssificans Progressiva Patients}

Introduction: Fibrodysplasia Ossificans Progressiva (FOP) is an extremely rare disease. Mutation of an autosomal dominant allele in the gene, known as Activin A Receptor type 1(ACVR1) causes the disease. The mutation causes fibrous tissue to be ossified spontaneously or when damaged. Inappropriate signaling by the mutated gene causes Bone Morphogenic Protein 4(BMP-4) to stimulate fibroproliferation of the mesenchymal stem cells, and provokes endochondral bone induction. This results in replacement of muscle and connective tissues into bones. The approach of Noggin gene therapy as a treatment helps to control heterotropic ossification by regulating the activity of BMP-4 in tissue. Objective: To develop an effective gene therapy that could inhibit BMP-4 induced heterotropic ossification. Materials and Methods: BMP-4 induced heterotopic ossification was used as model to test the ability of the BMP-4 antagonist, Noggin, to block de novo bone formation, either by local or systemic delivery. It duplicates the stages of FOP heterotopic ossification similar in human. Since Noggin acts locally, a Noggin mutein, hNOGDeltaB2, was engineered. It was shown to circulate systemically, and its ability to block heterotopic ossification was tested in mouse mode with the use of adenovirus-mediated somatic cell gene transfer. Results: BMP-4 induced mouse model developed heterotopic ossification. Local delivery of wild Noggin inhibited heterotopic ossification, but in systemic administration it was ineffective. Systemic delivery of adenovirus encoding hNOCDeltaB2 resulted in systemic levels that persisted approximately more than two weeks, which was sufficient enough to block BMP-4 induced heterotopic ossification. Conclusion: Delivery of Noggin mutien through gene therapy successfully inhibited the BMP-4 induced heterotropic ossification in mouse model. This is due to its mechanism, where Noggin is involved in controlling the process of ossification by regulating the concentrations of BMP-4 concentration in tissue through negative feedback mechanism.

14. An Audit of Compliance for the Investigation of Newly Detected M-Proteins and the Management of Monoclonal Gammopathy of Undetermined Significance Sugeeta Sukumar University of Manchester.

Introduction: Monoclonal gammopathy of undetermined significance (MGUS) is a benign cloncal cell disorder with a potential become malignant and requires monitoring depending on stratified risk. Aim. A clinical audit was carried out to assess two areas: firstly, identifying if patients are being appropriately referred and if a MGUS scoring system could be applied to improve referrals and predict malignancy; second part involves assessing the investigation and management of MGUS within the haematology department according to the risk stratified for each patient. Method. 204 patients with newly detected monoclonal proteins were identified over a 6-month period between July and December of 2015 using online databases called Quadramed and Evolve and analysed. Results Audit identified $19.4 \%$ of probable high-risk patients were not referred and the scoring system developed had a high predictive value in this small sample group. A new system has been implemented in the immunology department to prevent under diagnosis of patients. However, further education on MGUS risk stratification needs to be considered within primary care. Majority of guidelines are being complied with but there is a poor compliance in performing urine electrophoresis and computed tomography (CT) scans. With regard to management, 8 high risk patients have been discharged as they were deemed to be borderline scenarios and 6 low risk 
patients remain under follow up due to concomitant haematological conditions. Frequency of follow up needs to be standardized and carried out more frequently every 3---4 months which could be achieved using the telephone clinic service available.

15. Prevalence and Characteristics of Depressive Symptoms in University Students in Melaka, Malaysia: A Cross-sectional Study

Tan Jin Rong, Chan Ya Tze, Khoo Veejie, Laviniya Naidu, Muhammad Rafik

Faculty of Medicine, Melaka Manipal Medical College

Introduction: Depression is an emerging health problem, with an estimated 350 million people affected worldwide. Objectives: Our study aims to determine the prevalence and evaluate the characteristics of depressive symptoms among medical, law and pre-university. We perform an exploratory analysis to survey the relationships of depressive symptoms with several risk factors like gender, scholarship, housing and medical problems. Methods: An analytical cross sectional study was conducted on medical, law and pre-university students of two private institutions in Melaka Malaysia, between September and October 2014. A total of 376 students participated in this study $(M B B S=142, \operatorname{Law}=129$, Pre $\mathrm{U}=105$ ). They completed self- administered questionnaires which included socio-demographic questions and the Beck Depression Inventory (BDI) survey. Each item in the BDI can be further divided into 3 clusters: affective, cognitive and somatic. Statistical analysis was performed using post-hoc test for multiple comparisons and multiple logistic regression. Results: There were 162 (43.1\%) students with depressive symptoms (BDl>9). Law students were found to be more depressed with the highest mean BDI score (11.7 B.7) compared to MBBS (8.4 18.1$)$ and pre-university students (8.9+6.8). Affective, cognitive and somatic clusters were significantly higher among law students. For exploratory analysis of risk factors, law students and those staying in hostel were significantly more likely to develop depressive symptoms. Discussion: This is the first cross sectional study that directly evaluates the characteristics of depressive symptoms by applying clusters in Malaysia. Law students are more depressed might be due to law students' frequent fear of failure in an atmosphere of intensive competition, teaching method and emotionally abusive in law students. Students living in hostel alone are more likely to develop depression, this can be due to the loneliness they may be experiencing, as they have than been reported to be less happy, less satisfied and more pessimistic. Conclusion: There is high prevalence of depressive symptoms among law, medical and pre-university students. If detected early, students with depressive symptoms may be successfully managed with behavioral therapy, emotional support and interpersonal psychotherapy.

16. Randomized Controlled Trial Study: The Effect of Exercise on Reaction Time

Tan Jo Lynn, Ting Teck Hing, Nazrul Shahabudin, Nabila Aizzuddin, Saranya Kathirvel.

Community Medicine

Introduction: Reaction time (RT) is the elapsed time between the presentation of a sensory stimulus and the subsequent behavioural response. Physical exercise has been shown to develop or maintain mental at physical fitness and overall health. The objective of this experimental study is to assess the effect of short term exercise on reaction time among MMMC students. Methodology: This is a single-blinded RCT conducted on medical students from Melaka Manipal Medical College (MMMC) over an 8week period. Using block randomization, 60 participants aged 18 28 were randomized into 2 experimental groups who performed exercise of different intensities, and a control group. Prior to the intervention (exercise), each participant completed a selfadministered structured questionnaire. Blood pressure, heart rates, memory, reaction time were measured before and afte intervention. Collected data was processed in Microsoft Excel and analyzed using Epi-info 7 and SPSS. Descriptive statistics and
ANOVA were calculated. Results: There was a significant difference of Systolic Blood Pressure and Pulse Rate between intervention groups ( $\mathrm{p}<0.05)$. Changes in Reaction time, Diastolic BP and Memory were not significant $(p>0.05)$. Participant perception was measured using EFI scoring. Higher Total EFI and subscale scores indicate more positive mood states, while higher Physical Exhaustion scores indicate greater physical exhaustion. We found a significant difference in positive engagement and revitalisation ( $p<0.05)$, but no significance differences in tranquillity and physical exhaustion, $(p>0.05)$. Conclusions: Aerobic exercise of different intensities produces no improvements in reaction time. However, exercise improves mood. Exercise could help create a more enthusiastic, happy society. Exercise also helps in keeping the body refreshed and energetic, promoting a stress-free environment.

17. Glycemic Control among Adult Patients Attending Follow Up in Specialist Clinics, University of Malaya Medical Center and the Guideline Adherence in their Management Tan Yin Zhien

Introduction: The prevalence of diabetes mellitus has been on the rise in Malaysia. The Clinical Practice Guideline (CPG) for management of diabetes mellitus was updated to the latest version in 2009, but the guideline adherence in managing diabetes mellitus remained unknown. Objectives: To look into the co-morbidities, diabetic medications, glucose control, pill burden of the patients living with diabetes mellitus, the percentage of guideline adherence in terms of blood pressure control and prescription of statin among diabetic patients. Methods: A crosssectional study was conducted at the medical clinics of University of Malaya Medical Center, randomly recruiting 456 diabetic patients who has follow-up in the clinics in November and December 2014. Patients' demographic, co-morbidities and clinical information were gathered mainly via interview, while patients' medication and blood investigation results were retrieved from the medical records of the patients. Results: Dyslipidemia and hypertension are the common comorbidities associated with diabetes mellitus (80.3\% it $77.6 \%$ ). The drug combination with most number of patient is biguanide with insulin $(21.7 \%)$. Clucose control among the respondents are poor generally, with only $23.5 \%$ and $27.6 \%$ of the patients achieving target $\mathrm{HbA}_{1 C}$ and fasting plasma glucose levels respectively. Conducting a logistic regression using poorly controlled $\mathrm{HbA} 1 \mathrm{c}$ as dependent variable, variables that significantly contribute to the model are employment, triglyceride level, fasting plasma glucose and type of anti-diabetic treatment $(p<0.05)$. Blood pressure control is poor among the diabetics. Guideline adherence is good in the aspect of prescription of angiotensin converting enzyme inhibitor (ACEI), angiotensinreceptor blocker (ARB) and statins for hypertensive and dyslipidemia patients among the diabetic respondents. $46.3 \%$ of the patients are taking 6 to 11 pills daily (including aspirin and medication for diabetes, hypertension, dyslipidemia), with medication for other conditions not included. Conclusion: The glycemic and blood pressure control among the patients being followed-up in the medical clinics in UMMC remained poor. The guideline adherence in terms of prescription of ACEIs, ARBs and statins are good generally. More recommendations from the guidelines should be studied to obtain an overall picture of guideline adherence in managing diabetes mellitus.

\section{Perioperative Management of Patients with Cardiac Implantable Electronic Devices \\ Tharsini-Sarvanandan}

Introduction: Worldwide, several million people have received a cardiac implantable electronic device (CIED) as treatment for their arrhythmias. Although the introduction of these devices has completely transformed the treatment of patients who have cardiac conduction problems, it has made the management of these patients perioperatively more complex due to the presence of the device itself. Aim: This review aims to look at the basics of CIEDs, specifically, the various types and their function as well as current opinion and guidance on managing patients with these 
devices perioperatively. It also takes into consideration the potential complications that may arise and any interference these devices may be susceptible to in this critical period. Methods: A search was conducted through electronic databases including PubMed, EMBASE and Ovid. Keywords used in this search were 'pacemakers' $O R$ 'implantable cardioverter-defibrillator' $O R$ 'ICD' AND 'anaesthesia' OR 'perioperative'. The list of the search results was reviewed to determine the relevance to the topic at hand by screening the title and abstract of the papers. Results: In order to increase the probability of a good outcome, a thorough preoperative assessment as well as vigilant monitoring throughout this period is vital. Appropriate measures to ensure patient safety including reprogramming the device should be considered. Conclusion: The preoperative assessment is an opportunity to elicit information from the patient regarding their overall health and the function of their device so that the right preparations can be made if intraoperative problems are anticipated. During surgery, continuous monitoring is essential and equipment for resuscitation must always be readily available. Postoperatively, device function should be evaluated again.

19. Factors Associated with Differences in Haemoglobin Levels in Community-Dwelling older Individuals in the Klang Valley

Yew $S Y^{1}$, Bee $P C^{2}$, Tan $M P^{3,4}$

${ }^{1}$ Fourth Year Medical Student, ${ }^{2}$ Division of Haematology, ${ }^{3}$ Division

of Ceriatric Medicine, ${ }^{4}$ Ageing and Age-Associated Disorders Research Group, Department of Medicine, Faculty of Medicine, University of Malaya

Introduction: Anaemia is a significant independent contributor to morbidity, mortality and frailty in elderly patients, and can lead to cardiovascular and neurological events. It has a great impact on the quality of life. The objective of the study was to determine the factors associated with differences in haemoglobin level among the community-dwelling elderly in Klang Valley. Methods: Participants in this cross-sectional study were recruited from community-dwelling elderly ( 65 years and above) in Klang Valley during health awareness events. Anemia was defined according to World Health Organization(WHO) criteria as a hemoglobin ( $\mathrm{Hb}$ ) level of less than $13.0 \mathrm{~g} / \mathrm{dL}$ in men, and less than $12.0 \mathrm{~g} / \mathrm{dL}$ in women. Results: A total of 137 sampled residents met the criteria for data completeness and were retained for analysis. Mean age was $71.6+5.9$ years. Using WHO criteria, $9.5 \%$ of the participants were anaemic. Factors associated with differences in haemoglobin levels statistically $(p<0.05)$ were educational level of primary leve or less $(p=0.003)$ and age $>70$ years $(p=0.007)$. Hemoglobin level correlated significantly with weight $(r=0.406, p<0.001)$, height $(r=0.335, p<0.001)$ and $B M I(r=0.242, p=0.006)$, but negatively with age $(r=-0.231, p=0.007)$. Conclusion: Lower educational level, age lower body weight and BMI and lower height are associated with lower haemoglobin levels among community dwelling older residents in the Klang Valley. An understanding of risk factors associated with anaemia will help direct efforts to reduce the burden of anaemia in our population. 